\title{
How to manage robust tactical planning with an APS (Advanced Planning Systems)
}

\author{
Patrick Genin*, André Thomas**, Samir Lamouri* \\ * SUPMECA - LISMMA-OSIL (EA2336), 3 rue Fernand Hainaut 93407 SAINT-OUEN \\ Cedex, France \\ patrick.genin@supmeca.fr
}

**CRAN - Faculté des sciences - BP239 - 54506 - VANDOEUVRE les NANCY, France.

Andre.Thomas@cran.uhp-nancy.fr

\begin{abstract}
Nowadays, managing correctly the always changing customer demands is a challenge for companies, especially because of its impact on the Supply Chain (Forrester effect). Tactical planning is very useful in establishing robust plans. This paper proposes an alternative policy to traditional practices (frozen horizon ...), the so-called "reference plan", to obtain more stable and robust production plans at tactical level.

Using an industrial application and simulations, we illustrate how the different practices contribute to robustness in planning. The "reference plan" policy seems to realize the best compromise between stability, robustness costs and service levels achieved by the tactical plans.
\end{abstract}

\section{Key words}

Robustness, tactical planning, sales and operation planning, APS. 


\section{The Importance of the Robustness of Tactical Planning}

The persistent weaknesses encountered when using ERP systems have revealed the need for optimized tools to manage the logistics chain (Advanced Planning Systems: APS) (Ling, 2000). The problem lies in the knowledge of how to obtain maximum reactivity and flexibility at the tactical level (Sales and Operations planning - S\&OP, Master Production Schedule - MPS, Purchase and Distribution Plans) by ensuring the fulfilment of the global objectives defined in an optimal way at the strategic planning level. APS offer functions spread over three levels of management: strategic, tactical and operational. Broadly speaking, the strategic level enables the definition of the total capacities of the production units in the company's Supply chain (SC), namely: warehouses, markets etc... (Shobrys and White, 2000). The third level, named the operational level, handles problems of production activity control in the very short term.

The second level, Tactical Planning, and especially Sales and Operation Planning (S\&OP) makes it possible to implement the strategic objectives defined by "Senior Management" in the Business Plan, by defining the production tasks that must be carried out in order to achieve the global objectives. S\&OP leads to check whether the resources required, in terms of employee numbers, stock, investments, etc., are available on the factory floor. If this objective cannot be achieved, a decision will be taken to subcontract, for example. In industrial groups there is also the problem of the distribution of volumes between the various sites managed by the same sales team (Mukhopadhyay et al., 1998). All these calculations are carried out (often monthly) at the level of the family of products (Vollmann et al., 1997). S\&OP is entirely integrated into the commercial and information systems of demand management. It allows the various master production schedules to be put into practice (De Kok and Inderfurth, 1997) and to take a large variety of constraints into account in order to produce an optimized plan. 
Managers usually use frozen periods and time fences to stabilize production plans. Since some years, APS allow to use "dynamic horizons". APS allow for the frequent rescheduling of the various plans which exist at all levels of the decision-making throughout the supply chain. That leads to several changes in the S\&OP decisions, thus reducing the stability necessary for the plans at the operational level and throughout the supply chain. In this paper, we propose an alternative, named "reference plan", to manage the so-called robust tactical plan.

First, we present stability and robustness concepts. To introduce our proposition, we give an overview of the traditional practices for robust production plans. In the following parts, we develop an industrial application and we compare the different results obtained with traditional models, on the one hand, and with our model of "reference plan", on the other hand. Finally, we conclude and develop some perspectives.

\section{Stability and Robustness}

\subsection{Stability}

The term stability is generally thought of as being the opposite of nervousness. Nervousness is defined as the significant changes related to the quantities or the periods of the scheduled orders in MRP plans. These changes occur even in the event of tiny changes at a higher level or in the MPS (Vollmann et al., 1997). Since 1975, several researchers, including Orlicky (Orlicky, 1975), have noted the importance of the "nervousness" of MRP systems. In the literature, nervousness in planning is often addressed in the Master Production Schedule and the MRP levels (Blackburn et al., 1986, Yano and Carlson, 1987, Ho, 1989, Minifie and Davis, 1990, Sridaharan and Laforge, 1990, Jensen, 1993, Kadipasaoglu and Shriridharan, 1995, Heisig, 1998). For example, Kadipasaoglu and Sridharan show the difficulties induced by the nervousness because of uncertainty in demand, purchasing or in the dynamic calculation of lot size in MRP systems (Kadipasaoglu and Sridaharan, 1995). 
Several strategies have been proposed to reduce the nervousness or increase the stability of plans calculated using MRP systems:

- extend the planning horizon (Carlson, 1982),

- freeze the master schedule within the planning horizon (Zhao, 1993),

- $\quad$ ensure that buffer or safety stocks are in place (Blackburn et al., 1986),

- differentiate between large and small modifications (Ho, 1989).

In their article, De Kok and Inderfurth study the plan's stability in quantities, i.e. the difference of planned quantities in each time bucket between two calculations (De Kok and Inderfurth, 1997). Like Kimms (Kimms, 1998), they use the expected value of quantities variations between two planning periods since their model is stochastic. Donselaar et al. compare the nervousness of the plan generated by MRP with that of their heuristic plan (Donselaar et al., 2000). The indicator used is the number of "reschedulings" carried out. Following Lyapunov (1892), we define a schedule as being stable if and only if the number of modifications in the levels of the decision variables between two successive establishments remains within a level considered to be convenient for the manager.

Stability is thus related to the decision variables in the tactical plan.

\subsection{Robustness}

Robustness studies and some approaches have been proposed in several areas such as quality management, manufacturing design (Lim et al., 1996; Durieux and Pierreval, 2003), scheduling (Davenport and Beck, 2000; Artigues et al., 2005), control policies of production system (Kleijnen and Gaury, 2003) or operational design of supply chain (Shang et al., 2004). The term robustness is generally associated with those two terms risk and decision-making (Kleijnen and Gaury, 2003). G. Taguchi analyses the robustness of a function by making the assumption that it can be controlled by the signal/noise ratio that combines the mean and the standard deviation of the result (Taguchi, 1987). 
There are several definitions of robustness. For Lee et al., robustness relates to the results of one or more functions and their dispersion due to uncertain parameters and costs (Lee and $\mathrm{Yu}$, 1997). In a very different context from ours, Kleijnen and Gaury study the robustness of a kanban loop according to two functions: the expected value of the work-in-process of the loop and the delivery rate (Kleijnen and Gaury, 2003). Zäpfel (Zäpfel, 1998) and Roy (Roy, 1998) use another definition: a tactical plan is robust if it allows an achievable plan to be found for the problem of operational planning for all possible outcomes of the demand.

All these definitions consider robustness as either an attribute of a plan or as a property observed following the changes which occur through the course of time. We prefer to follow the latter definition in order to be consistent with the given definition of stability. We qualify planning as being robust if and only if its characteristics show a weak dispersion in spite of the disruptive fluctuations of noise factors.

Robustness is thus related to the dispersion of one or more performance measurements.

Thus, a system can be robust, i.e. showing a weak dispersion of the measured functions, while being unstable: the manager changes systematically his decisions variables (instability) to reach the objective value (robustness). At the opposite, a system can be stable, i.e. generating little variation in the decision variables, while being not robust: the measured function diverges (lack of robustness) whereas the decisions variables are unchanged (stability).

\section{Traditional management of Stability}

\subsection{Planning Time Fences}

The principal approach for stabilizing plans consists of reducing the modifications that are made. Stability is introduced by devices such as planning time fences and frozen horizons. A frozen horizon of $\mathrm{X}$ weeks means that no modifications are possible within these $\mathrm{X}$ weeks. In practice, the term "no modifications" is somewhat strong. Companies have scant regard for 
the term "frozen". They consider that everything is negotiable. But negotiations become more difficult the nearer one gets to the present moment. (Vollmann et al., 1997).

The APICS dictionary defines a planning time fence as a policy or guideline established to note where various restrictions or changes in operating procedures take place (APICS, 1997). The impact of time fences on the stability of plans is well known (Zhao and Lee, 2003), but the impact of this mode of management on the robustness of tactical planning does not seem to have been studied. We will examine this issue in our work.

\subsection{Management by exception supported by the APS}

Ho differentiates between the use of large and small modifications for stabilizing plans (Ho, 1989). The mode of management by exception leads to the management of exceptional events in the APS: an unexpected sale, significant changes in customer orders, the breakdown of a key machine, etc. A new plan is established, not at regular intervals, but in the event of consequent modifications. This procedure is often presented as being more effective (Stadtler and Kilger, 2000) and is thus used by companies having an APS.

This mode of management makes it possible to make the frequencies of planning "dynamic" by creating the reactivity that is necessary for any supply chain. However, just as for the planning horizons, the analysis of the impact of this mode of management on the robustness of the plans does not, to our knowledge, seem to have been studied. Our work will show the benefits of such an approach.

\subsection{Sensitivity Analysis}

APS create plans by using a linear or a holistic modeling of tactical planning problems. Sensitivity analysis is the tool usually proposed to answer the question of the stability or of the robustness of decisions arrived at by the resolution of linear programs. Koltai and Terlaky highlight the differences in interpretations between the sensitivity of management and the mathematical analysis of sensitivity (Koltai and Terlaky, 2000). 
The objective of sensitivity analysis is to study the effect of a change in the coefficients of the objective function and the effect of a change in the values of the constraints on the optimal value of the objective function, as well as the ranges of validity of these effects. The analysis of sensitivity gives information on the interval of values of a parameter for which the solution remains optimal, and the rate of variation of the objective function. With regard to the problem of decision, the software should provide the information easily, which is seldom the case with APS when used to provide an analysis of sensitivity.

Two criticisms can be made regarding sensitivity analysis: (1) this analysis, which is calculated in order to provide a solution, is carried out post-optimization. It is not used as criterion of optimization in the linear programming model and does not lead the model towards a robust solution; (2) the exact result of a linear programming model is sometimes not easily exploitable because its application requires a major reevaluation of the existing situation. Next, it is interesting to compare the behavior of the various optimal solutions. The software could be used to illustrate them, by showing the invariants and the alternatives in the decisions. To complete our criticism of the analysis of sensitivity, it is significant to note that it works by acting on a parameter-by-parameter basis. It does not consider the effect of the interactions, and yet when several constraints exist, their simultaneous analysis is essential. In this article, we propose a method of tactical planning management, leading to robust decisionmaking capacity within the framework of use of APS. In this context, we have taken as our hypothesis the fact that APS apply the method of resolution by linear programming.

\subsection{Synthesis}

Because of the changeable nature of the tactical plan, the relevance of a decision taken over one particular period of time can only be judged after several periods have elapsed. The simulation of an operating mode, that is flexible on the tactical planning level, thus appeared to be a sensible approach to take. In what follows, we propose to compare the three main tactical planning processes: (i) Frozen periods with time fences, (ii) "dynamic frozen 
horizon", (iii) and the new approach that we named "reference plan". We illustrate, by means of an industrial application, how to obtain greater robustness in planning through these different practices.

\section{Explanatory Notes for the Presentation of the Test-Case}

The model presented is simplified: we are considering only one production line and only one family of products. Furthermore, this model of linear programming can be regarded as benchmark for our experiments.

\subsection{Notation}

$\underline{\text { Indices }}$

$h$ : horizon of the tactical plan,

$t$ : index of the period of the plan $t=1,2 \ldots, h, t=0$ indicates the initial conditions.

$\underline{\text { Parameters }}$

$F_{t}$ : forecasted demand of the period $t$ (Table 1),

$u_{t}^{P}$ : quantity of a unit of production in period t per operator,

$u_{t}^{H}$ : quantity of a unit of production by overtime in period $t$

$C_{t}^{R}$ : maximal available machine capacity in period $t$ in units of production,

$C_{t}^{s}$ : maximal available storage capacity in period $t$ in units of production,

$C_{t}^{H}$ : number of additional overtime hours authorized by operator in period $t$,

$s_{h}:$ stock target at the end of the planning horizon,

$r_{h}$ : delay target at the end of the planning horizon,

$o_{h}$ : operators' target at the end of the planning horizon.

$\underline{\text { Variables }}$ (Table 2) 
$S_{t}$ : stock of finished products at the end of

period $t$ (positive or zero),

$R_{t}$ : shortages existing in finished products

at the end of period $t$ (positive or zero),

$S_{t}^{M}$ : stock of raw materials at the end of

period $t$ (positive or zero),

$O_{t}$ : number of operators in period $t$

(positive or zero),

$H_{t}$ : number of overtime hours in period $t$,

\section{$\underline{\text { Costs }}$ (Table 1)}

$c_{t}^{S}:$ cost of storage per unit of production in

period $t$,

$c_{t}^{S M}$ : cost of storage per unit of raw

materials in period $t$,

$c_{t}^{R}$ : cost of delay per unit of production in

period $t$,

$c_{t}^{E}$ : unit cost of recruiting employees in

period $t$,
$E_{t}$ : planned number of employees to be

recruited in period $t$,

$L_{t}:$ number of layoff planned for in period

$t$

$P_{t}:$ number of production units manufactured in period $t$, $M_{t}$ : number of units of materials to be procured in period $t$.

$c_{t}^{L}:$ unit cost of redundancies in period $t$,

$c_{t}^{O}$ : cost per operator in period $t$ (wages),

$c_{t}^{H}$ : cost of one overtime hour in period $t$,

$c_{t}^{A}$ : unit cost of raw materials procured in period $t$,

$c_{t}^{T}$ : total cost of the plan at period $t$,

$c^{T}$ : total cost of the plan.

\subsection{Values of the parameters}

Tactical plan calculated on a twelve months horizon: $h=12$. The different data used for the simulations are given in tables 1 and 2 .

\section{TABLE 1}

TABLE2 


\subsection{Indexing of the model}

The following notations have been introduced for the purposes of the simulation:

$n$ : number of periods incremented during the simulation

$p$ : current period of the simulation $p=1,2 \ldots, \mathrm{n}$

$t$ : index of the period of plan $\mathrm{t}=p, p+1 \ldots, p^{+h-1}$

$D_{p}:$ actual demand of period $\mathrm{p}$,

$F_{p t}$ : forecasted demand for period $t$ established at the beginning of period $p$.

\subsection{The model}

The manager seeks to optimize the use of resources while minimizing the total costs of the plan over the whole planning horizon $c^{T}$, under the constraints of the equations 2-10.

$c_{p}^{T}=\sum_{t=1}^{h} c_{p t}^{S} \times S_{p t}+c_{p t}^{R} \times R_{p t}+c_{p t}^{E} \times E_{p t}+c_{p t}^{L} \times L_{p t}+c_{p t}^{O} \times O_{p t}+c_{p t}^{H} \times H_{p t}+c_{p t}^{S M} \times S_{p t}^{M}+c_{p t}^{A} \times M_{p t} \forall p(1)$

These costs are made up of: inventory holding costs, backorder and stockout costs, hiring and layoff costs, labor costs, overtime costs, inventory holding cost of the raw materials, cost of procuring materials. The constraints of the model are:

Stock constraints: $\begin{aligned} & S_{p, t-1}-R_{p, t-1}+P_{p t}=S_{p t}-R_{p t}+F_{p t} \quad \forall t, p \\ & S_{p, t-1}^{M}+M_{p t}=S_{p t}^{M}+P_{p t} \quad \forall t, p\end{aligned}$

Materials availability: Production can only be processed with materials in stock during the preceding period; therefore, we have created a procurement delay for one period: $P_{p t} \leq S_{p, t-1}^{M} \quad \forall t, p$

Operator constraints: $O_{p, t-1}+E_{p t}-L_{p t}=O_{p t} \quad \forall t, p$

Targets for stocks, delays and number of operators: $\begin{aligned} & S_{p, p+h-1}=s_{p h} \\ & R_{p+h-1}=r_{p h} \quad \forall p \\ & O_{p+h-1}=o_{p h}\end{aligned} \quad \forall$

Constraint of machine capacity: $P_{p t} \leq C_{p t}^{R} \quad \forall t, p$

Constraint of labor capacity: $P_{p t} \leq u_{p t}^{P} \times O_{p t}+u_{p t}^{H} \times H_{p t} \quad \forall t, p$ 
Storage constraint: $S_{p t}+S_{p t}^{M} \leq C_{p t}^{S} \quad \forall t, p$

Overtime constraint: $H_{p t} \leq C_{p t}^{H} \times O_{p t} \quad \forall t, p$

Constraint of positivity of variables: $0 \leq S_{p t}, R_{p t}, S_{p t}^{M}, O_{p t}, H_{p t}, E_{p t}, L_{p t}, P_{p t}, M_{p t} \quad \forall t, p$

\section{Experimental approach}

We are seeking to validate the impact of the management methods that are applicable to APS and to analyze their effects on the robustness of the tactical plan. Not so far in the past, tactical planning decisions, such as the installation of a new team, or new investment, required an irreducible time for their implementation, which established a genuine frozen horizon. Nowadays, the flexibility acquired makes possible the application of decisions on a month-by-month basis. It becomes possible for a decision to be made over one month and changed over the next. The manager can adopt one of the three following management methods:

1. reoptimize the planning model at each period in order to be permanently in phase with demand and to have a permanent access to an "ideal" plan. The frequency of rescheduling constitutes the frozen horizon,

2. preserve a frozen horizon, allowing for the stabilization of production and procurement,

3. reoptimize only if the situation deviates significantly from the plan, when an indicator triggers an alarm. The frozen horizon is then considered to be "dynamic".

The first experiment is concerned with a situation in which the plan is reoptimized at each period. The second considers a frozen time fence of three periods and the third simulates a "dynamic" horizon where an indicator is evaluated at each sub-period. In our case, this third solution means that the manager reoptimizes the model if the requested demand deviates 
significantly from the forecast and if it exceeds the established tolerances. The limitations of these approaches have led us to propose a new practice: the use of a reference plan, which we will develop further, in $\S 6.3$

\subsection{Logic of the simulation}

For each management method tested, the following stages were performed:

1. Initialization $t=0$ with the initial forecasts;

2. Establishment of the plan for the period $p$ : optimization of the model over $h$ periods;

3. Implementation of the plan over the period $p$ : indication of the demand; $D_{p}$

4. Evaluation of the actual costs of the part of the plan $n$ in period $p: c_{p p}^{*}$ cost of the current period $p$ for the demand; $D_{p}$

5. Calculation of the forecasts for the period $p+1 ; F_{p+1, t}$

\subsection{Establishing the actual demand}

In their article, Disney et al. analyze the impact of an original approach to inventory control in a supply chain on the Forester effect (Disney and Towill, 2003). They measure the variations in the behavior of the objective function of their simulation model when compared with changes in the volume of demand. For this, the demand was modeled by a normal law of average 0 and standard deviation 1 . In a different approach, Rota et al., modeled the projected demands according as a uniform random number between minimum and maximum bounds (Rota et al., 2002).

In our case, using the same experimental logic as used by the latter, the demand actually consummated, $D_{p}$, is established from the estimated demand from period $F_{p p}$, to which we add a bias $\mathrm{B}_{\mathrm{p}}$ (Equation 11). This bias is a random number that follows a normal distribution of average 0 and standard deviation 6 with an upward trend of $6 . D_{p}=F_{p p}+B_{p} \quad \forall p$ 
The combination of the model of forecasting by first-order smoothing (see hereafter) and the method of generation of demand, makes it possible to simulate situations in which the demand is not entirely random. The nearer one gets to the time of the month, when decisions are implemented, the better is the forecast, which is often the case in practice. The variation in volume of the demand constitutes the disrupting factor for the system that we have simulated. However, in a supply chain, uncertainty is apparent through demand, process and procurement (Zäpfel, 1998) and (Roy, 1998).

\subsection{Evaluation of Actual Costs}

The decisions implemented in period $p$ are:

- volume of production $P_{p p}$

- decisions regarding hiring of employees/redundancies $E_{p p}$ and $L_{p p}$,

- number of operators $O_{p p}$

- number of overtime hours allocated $H_{p p}$,

- quantity of raw materials procured $M_{p p}$.

The variables influenced by the difference between the forecast for the period $p, F_{p p}$ and the actual demand $D_{p p}$ are the levels of stock, $S_{p p}$ and of delay, $R_{p p}$ bound by the equation (2) where the projected demand, $F_{p t}$ is replaced by the demand $D_{p}$ :

$S_{p, p-1}-R_{p, p-1}+P_{p p}=S_{p p}^{*}-R_{p p}^{*}+D_{p} \quad \forall p$

Once the volume of the actual demand $D_{p}$ is known, the costs of the part of the plan implemented $c_{p}^{*}$, can be determined by equation 13 :

$c_{p}^{*}=\begin{gathered}c_{p p}^{S} \times S_{p p}^{*}+c_{p p}^{R} \times R_{p p}^{*}+c_{p p}^{E} \times E_{p p}+c_{p p}^{L} \times L_{p p} \\ +c_{p p}^{O} \times O_{p p}+c_{p p}^{H} \times H_{p p}+c_{p p}^{S M} \times S_{p p}^{M}+c_{p p}^{A} \times M_{p p}\end{gathered} \quad \forall p$

This cost is saved after each iteration of the simulation to give the total cost generated $c^{*}$, using:

$c^{*}=\sum_{p=1}^{n} c_{p}^{*}$ 


\subsection{Establishing the forecasts}

Since we are seeking to study the robustness of the various practices, we must create a sufficient degree of uncertainty in order to generate some variability. Vollmann et al. indicate that low values of first order smoothing. $(\alpha=0.1)$ lead to stability in the forecasts faced with the random behaviors of the demand, but these values lead also to slow reactions to changes in trends of the demand (Vollmann et al., 1997). On the contrary, high values of $\alpha$ give more weight to recent demand and therefore provide more sensitive forecasts for the behaviors of the demand. This phenomenon is also explained by Lee et al. (Lee et al., 1997) and by Simanpo (Simanpo and Ryan, 2003). The forecasts are established by first-order smoothing.

$$
\begin{aligned}
& F_{p+1, p+1}=\alpha D_{p}+(1-\alpha) F_{p, p+1} \quad \forall p \\
& F_{p+1, t}=\alpha F_{p+1, t-1}+(1-\alpha) F_{p, t} \quad \forall p, t \quad p+1<t<p+h \\
& F_{p+1, p+h}=\alpha F_{p+1, p+h-1}+(1-\alpha) D_{p} \quad \forall p
\end{aligned}
$$

The smoothing constant $\alpha$ was fixed at 0.3 . By fixing the constant at this level, the forecasts are sensitive to changes in the demand. This creates the required volatility. This hypothesis is, of course, a necessary requirement for the simulations. In order to keep the seasonality, the last equation calculates the forecast for the period entering into the rolling planning horizon, as part of the demand $D_{p}$ of the current period that is coming out of the planning horizon.

\subsection{Establishing the plan for the period $p$}

Once the forecasts have been calculated, the index of period $p$ is incremented. The model is re-initialized by redefining the parameters:

$$
\begin{aligned}
& S_{p, p}^{*} \rightarrow S_{p+1, p}, \\
& R_{p, p}^{*} \rightarrow R_{p+1, p}, \\
& O_{p, p} \rightarrow O_{p+1, p}, \\
& p+1 \rightarrow p .
\end{aligned}
$$

These definitions initialize the initial levels of stock and delay and the initial number of operators for the new plan $p+1$. The deterministic model is reoptimized on the rolling 
planning horizon of $h$ periods, taking the new forecasts into account. The iterations are performed $n$ times.

\subsection{Performing the Simulations}

The simulations were carried out using:

- Excel 2000 and Visual BASIC for Applications 6.0 in order to (1) apply the logic of simulation; (2) generate the random demand. (3) describe the deterministic model.

- LP-TOOLKIT for Visual BASIC to optimize the linear model. This code was developed by Euro-Decision $(\subset)$ in partnership with Dash Optimization by using XPRESS MP technology.

For each experiment, 10’000 simulations were carried out on a Pentium III/1Ghz personal computer with $256 \mathrm{MB}$ of RAM.

\subsection{Indicators Used}

Because of its importance, the performance indicator that we retained on grounds of its robustness is the cost of the tactical plan implemented, $c^{*}$ (equation 14). Here, we would also like to point out that the tactical plan is often developed on the basis of an economic unit of work, because this is the only one which allows the influences of different actions to be compared. It also constitutes one of the objectives of the tactical plan. This cost corresponds to the cost of the first period of the plan, once the demand is known and the decisions of the first period have been implemented.

The indicator of robustness used is the dispersion around its average of the real cost of the plan in operation over one rolling year. It will be measured by the standard deviation of the total costs obtained on the simulations.

The indicator of stability that we use is different from that chosen by De Kok (De Kok and Inderfurth, 1997) or by Kimms (Kimms, 1998). In our case, the indicator used to measure 
instability, Is, relates to the variation in volume between the procurement forecast for the period $t$ predicted in $t-1$, and the actual quantity procured in period $t$ (Formula 16).

$$
\begin{aligned}
& \text { if } M_{p}(p) \neq M_{p-1}(p) \text { then } \\
& \text { Is }=I_{s}+1 \forall p
\end{aligned}
$$

In addition to the indicators of robustness and stability, the service level will be evaluated.

$I_{p}^{T S}$ is the service level of period $p$; it is the relationship between the quantity delivered over period $p$ and the quantity to be delivered for $p$. The quantity to be delivered for $p$ is the sum of the delay from the previous period $p-1$ and of the demand from period $p$. The overall service level $I^{T S}$, is the average of $I_{p}^{T S}$ over the n periods simulated (Formula 17).

$$
\begin{aligned}
I_{p}^{T S} & =\frac{R_{p, p-1}+D_{p p}-R_{p p}}{R_{p, p-1}+D_{p p}} \forall p \\
I^{T S} & =\underset{p=1}{n} e^{n}\left(I_{p}^{T S}\right)
\end{aligned}
$$

\section{Experiments and results}

\subsection{The case with planning time fences}

A frozen horizon of $\mathrm{f}$ periods was modeled. This horizon means that the decision variables in the frozen horizon should not be changed during these periods.

f: number of periods of the frozen horizon.

In the optimized model, some additional constraints were added to represent the fixing of the decision variables:

- the level of production remains unchanged over the $f$ periods:

$P_{p t}=P_{p-1, t} \quad \forall p, t \quad p \leq t<p+f$

- the number of operators is fixed for $\mathrm{f}$ periods:

$$
\begin{array}{lll}
E_{p t}=E_{p-1, t} & \forall p, t & p \leq t<p+f \\
L_{p t}=L_{p-1, t} & \forall p, t & p \leq t<p+f \\
O_{p t}=O_{p-1, t} & \forall p, t & p \leq t<p+f
\end{array}
$$


- the overtime hours were decided over f periods:

$$
H_{p t}=H_{p-1, t} \quad \forall p, t \quad p \leq t<p+f
$$

Optimization will establish the resulting levels of stock or delay for each of the frozen periods.

The stages of simulations remain unchanged. The frozen horizon, $f$, is fixed at 3 for the experiment.

\subsection{The case using a "dynamic" horizon}

In this case, an optimization of the whole of the plan is carried out only if an indicator reaches a predetermined threshold. In our model, the only existing uncertainty is the demand, leading to a variation in the levels of stock or delay. In practice, in order to be consistent with the forecasting model used, managers use the difference between the forecast and demand: the error (or bias) (Vollmann et al., 1997). This indicator will trigger the alarm. The model previously described was modified to evaluate this indicator at more frequent intervals than at each period. Each of the first four periods of the planning horizon was divided into four subperiods, giving sixteen sub-periods. The remaining periods of the planning horizon were left unchanged. Consequently, the forecasted demands $F_{p t}$ established for the periods were disaggregated linearly over the four sub-periods. The capacities of the resources and storage, $C_{t}^{R}$ et $C_{t}^{S}$, the quantity of production per period, $u_{t}$, the number of authorized overtime hours, $C_{t}^{H}$ and the costs relating to the delays and storage, were also disaggregated linearly.

To allow comparison of the results, the indices were also changed:

$-n \rightarrow 4 n$

$-h \rightarrow 1 \ldots 16,5 \ldots h$

- $p$ and $t$ respectively describe the index of the sub-period of simulation and the index of the sub-period considered on the planning horizon.

The actual demand for each sub-period is obtained by equation $11 . B_{p}$ follows a normal distribution of average 0 and standard deviation 3. This standard deviation is obtained by the 
equation. $\frac{\text { Standard Deviation }}{\sqrt{\text { number of sub - periods }}}=\frac{6}{\sqrt{4}}=3$. This equation determines the standard deviation of the sub-period in order to guarantee the standard deviation of the period in the case of a normal distribution. The trend is also divided by 4 . The simulation stages were modified. The indicator triggering a reoptimization of the model is the difference between the projected demand of the sub-period and the actual demand of the current sub-period.

- If the absolute value of this difference is higher than 2 standard deviations, an optimization of the whole planning horizon is carried out (as in the case without a horizon). This number is selected arbitrarily. It has to result in the plan being reviewed only rarely because of the high level of dispersion of demand that can be reached before the alarm is triggered.

- If this does not happen, the index of simulation is incremented. In order to absorb the variation in the demand, an optimization is carried out over the first four sub-periods, with the aim of reaching the level of stock/delay and number of operators for the fifth subperiod (the aim of the MPS is the achievement of its monthly objectives). If the sub-period is the first of a new period, optimization is carried out only over the eight previous periods (the SOP is then re-examined, taking a frozen horizon into consideration).

This procedure represents a situation in which events are absorbed by the decisions of operational management, in order to avoid forcing a reappraisal of the entire tactical plan. It respects the logic of hierarchical planning in which the lower level of planning seeks to achieve the volumes defined at the higher level of planning.

Initially, we carried out experiments for the three traditional management methods. These made it possible to highlight the strengths and weaknesses both of the existing practices, (management with or without planning time fences) and of the emergent methods (management by exception). Here, our concern is to merely summarize the findings. For a detailed development, we refer the reader to the work of Genin (Genin 2003). However, these 
simulations have enabled us to propose a new mode of operation: management by reference plan.

\subsection{Reference plan: the role of stability in promoting robustness}

\subsubsection{Introduction of the concept}

The plan optimized in period $p$ is called $P_{p}^{O}$. However, some decisions could already have been taken and implemented. Their effects will be perceptible in later periods. This plan in progress, started previously, is called. $P_{p}^{E}$. Differences can exist between these plans according to events that have occurred. Traditionally, the manager modifies the plan $P_{p}^{O}$ in the light of plan $P_{p}^{E}$ in order to find an intermediate solution, which takes account of not only the new conditions, but also of decisions taken previously. This compromise plan for the period $p P_{p}^{R}$, is called the "reference plan". It serves as a framework to help the manager develop the tactical plan for the new period $p+1$. If the modifications are considered to be not very significant, the reference plan is renewed for the following period. If not, it is calculated as a compromise between the reference plan for the previous period and the optimized plan without reference to the preceding decisions.

\subsubsection{Modification of the model}

In this experiment, the modeling of the decision-making situation from the preceding experiments is modified to include the development of a reference plan, $P_{p}^{R}$ in the planning process. The manager's aversion to the modifications of these decisions is represented by a weight on the modifications, i.e. on the difference between the new decision variables and those of the reference plan. The model seeks consequently a compromise between the cost induced by this difference and the cost of the new "ideal" plan.

We chose the estimated procurement quantities that are transmitted to the supplier as the decision variables to stabilize. Indeed, one of the main priorities of tactical planning in practice is to stabilize raw materials quantities in order to limit the spread of variability. The 
procurement plan remains the reference plan as long as the control factors that influence it do not change significantly.

\subsubsection{Linear model with reference plan}

In order to model this mode of decision-making, the following notations are introduced:

$c_{p t}^{X}$ : unit overcost, in period $p$ for the period $t$, of purchasing raw materials in relation to the reference plan,

$c_{p t}^{N}$ : unit overcost, in period $p$ for the period $t$, for the cancellation of raw materials in relation to the reference plan,

$X_{p t}$ : number of units of materials supplied in addition to the reference quantity in period $t$ which were planned in period $p$,

$N_{p t}:$ number of units of materials cancelled in period $t$ which were planned in period $p$.

$X_{p t}$ and $N_{p t}$ represent the deviation from the reference plan. A deviation exists if one of these variables is positive. These additional variables are determined by optimization of the linear model. This optimization consists of seeking a compromise between stability (zero $X_{p t}$ and zero $\left.N_{p t}\right)$ and the costs of delays.

The objective of the planning remains the same: to minimize the costs over the planning horizon. However, the costs of cancellation or additional purchases are taken into account when the plan is being developed. These represent the manager's willingness or aversion for undertaking these modifications.

The optimized cost function becomes:

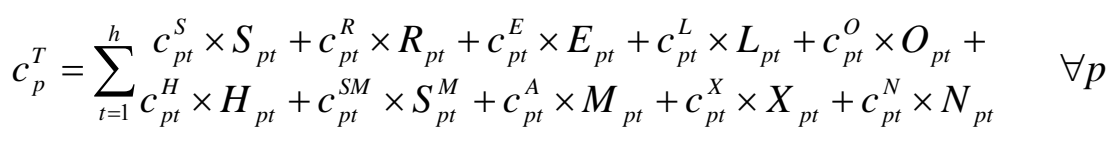

It is optimized under the constraints of the equations 2-10 and 22-23:

Relationship of the addition or removal of raw materials: $M_{p t}=M_{p-1, t}-N_{p t}+X_{p t} \quad \forall p, t$

Constraint of positivity of the variables: $0 \leq X_{p t}, N_{p t} \quad \forall p, t$ 


\subsubsection{Simulation}

For each of the cases, the $10^{\prime} 000$ simulations proceed in an identical way. In the case of simulation with a reference plan, the procurement plan for the period that is finishing is saved so that it can be used as input data in the model for the following period.

\subsubsection{Results for the reference plan method}

We note a reduction in the costs, but also in the standard deviation, indicating an increase in robustness. The costs decrease by $3 \%$ and the standard deviation by $7 \%$ (Table 3 ). Management of planning with a reference plan leads to lower annual costs, while improving the robustness of raising the costs. There is a major difference in the service level indicator $I^{T S}$ (Table 3). The strategy with a reference plan results in an increase of more than $9 \%$ compared to the basic case. This result is obtained in this case by establishing a safety stock of raw material. Indeed, the model favors the stocking of raw materials, even if it means incurring high storage costs. This makes it possible to react more quickly to unforeseen demand without risking modification costs.

With regard to the indicator of stability, $I_{S}$, the advantage with the "reference plan" strategy remains significant when compared to the basic case (Table 3). Thus, with this type of model, one gains a degree of freedom that is not possible using the frozen horizons which are traditionally used to reduce nervousness.

\subsection{Summary of the results}

In order to summarize the findings, we have included the costs bound to the modifications of the reference plan in order to allow comparisons to be made between the simulations of the traditional methods of management (with time fences and dynamic horizons). Figure 1 presents the distribution of costs. 
The mode of management with planning time fences leads to greater robustness in the plans and a reduction in the average costs. In this regard, it is the most effective strategy. The reference plan has an identical but less pronounced effect. The method of management by dynamic horizon and the reference method have practically the same effect on the costs, causing them to increase because of the modifications made to the reference plans,_and in this respect they are strategies to avoid.

On the other hand, the indicator of robustness, the standard deviation, is different for each of the management methods used. The dynamic horizon, the base case and the method of management with a reference plan lead to a certain robustness in planning. But the method with frozen horizons results in plans less robust because of its inertia to modifications.

\section{FIGURE 2}

Figure 2 shows the service level indicator, $I^{T S}$. In our simulations, the inertia of the method of management with planning time fences leads to a strongly diminished service level. The methods of management by dynamic horizon and by reference plan make it possible to be regularly in step with demand, which enables them to ensure excellent service levels. It is interesting to observe that the mode of management with a dynamic horizon leads to excellent service levels on some simulations. This is ensured by the iterations over the first sub-periods. The basic case is situated at an intermediate level.

\section{FIGURE 3}

Figure 3 shows the indicator of stability. We have not shown the curve for the method of management by dynamic horizon (calculated by dividing the number by 4), because the basis for comparison is not identical: in this management method, the procurement plan is potentially reviewed at each sub-period, whereas the other methods only revalue it at each period.

\section{TABLE 3}

Table 3 presents a quantitative summary of the indicators for each mode of management. 
The conclusions of this study could be influenced by the parameters used in the experiments. However the conclusions obtained for the traditional policies are on line with the results met in practice or checked by others researches. Although we did not realize an extensive experiment design, we made the same experiments with different sets of parameters. All of them give the same ranking of the policies.

\section{Conclusion}

The results are as expected for the first three experiments: (1) planning time fences reduce reactivity (complete stability and low service levels) and lead to low costs when they include the costs of modifications; (2) the cost increases for management by exception, but so does robustness (reduction in the standard deviation); the same can be said for reactivity (increase in service levels), while preserving stability.

This work could be further developed in order to model not only one fixed area, but also a controlled area in which certain decisions could be still contemplated. With regard to the chosen method of modelling, it seems that the method of management with planning time fences is the best adapted for minimizing the generation of variability upstream of the supply chain and thus for reducing the costs of the tactical plan. However, this strategy is strongly disadvantaged by its low service levels and lack of robustness, with a large range of variation. The method of management by exception ensures a better service level while improving robustness, but to the detriment of costs (equivalent to the costs of the basic case) and stability. The method of management by reference plan therefore achieves a good compromise between these two approaches: low costs, excellent stability, good robustness and an intermediate service level. The reference plan also allows the range of cost variations to be considerably reduced, thus lessening the risk taken by the manager. 
It might be interesting to combine these last two approaches in order to accentuate their positive effects. This represents a potential area for further study. An other important perspective lies in the objective to keep the obtained stability and robustness SOP characteristics at the desegregated Master Production Schedule level. Relying on Bitran and Hax desegregation models (Bitran \& Hax, 1977), we will verify if qualities of SOP are conserved at the Master production schedule level (Ortiz \& Thomas, 2005). Several approaches are possible, i.e. fuzzy logic (Toly, 2003) or stochastic approach (Suhua and all, 2000). It will be interesting then to study the recent research work of Miller (Miller, 2004) concerning the application of their initial results on the specific case of the Supply Chain.

\section{References}

ARTIGUES, C., BILLAUT, J.C., ESSWEIN, C. (2005) Maximization of solution flexibility for robust shop scheduling. European Journal of Operational Research, 165(2), 314--328 BITRAN, G.R. and HAX A.C. (1977) On the design of hierarchical production planning systems. Decision Science, 8, 28--55.

BLACKBURN J.D., KROPP H.D., MILLEN R.A. (1986) Comparison of strategies to dampen nervousness in MRP systems. Management Science, 32 (4), 413--429.

CARLSON R.C., BECKMAN S.L., KROPP D.H. (1982) The effectiveness of extending the horizon in rolling production schedules. Decision Sciences 13 (1), 129--146.

DAVENPORT, A.J., BECK, J.C. (2000) A survey of techniques for scheduling with uncertainty. Available online at: www-poleia.lip6.fr/ sourd/gotha/index.html (accessed 01 January 2000)

DE KOK A.G., INDERFURTH K. (1997) Nervousness in inventory management: comparison of basic control rules. European Journal of Operational Research, 103, 55--82. 
DISNEY S.M., TOWILL D.R. (2003) The effect of vendor managed inventory (VMI) dynamics on the Bullwhip Effect in Supply Chains. International Journal of Production Economics, 199--215.

DONSELAAR K.H.V., NIEUWENHOFJ.V.D., VISSCHERS J. (2000) The impact of material coordination concepts on planning stability in supply chains. International Journal of Production Economics, 68, 169-176.

DURIEUX, S., PIERREVAL, H. (2003) Comparison of several design solutions of a real industrial manufacturing system using simulation and risk analysis. Industrial Engineering and Production Management conference, 2, 145--154.

GENIN P., 2003, Planification tactique robuste avec l'usage d'un APS ; proposition d'un mode de gestion par plan de référence, Ph-D thesis Ecole des Mines de Paris, Décembre 2003. HEISIG G. (1998) Planning stability under (s, S) inventory control rules. OR Spektrum, 20 (4), 215--228.

HO C. (1989) Evaluating the impact of operating environments on MRP system nervousness. International Journal of Production Research, 27, 1115--1135.

JENSEN T. (1993) Measuring and improving planning stability of reorder point lot-sizing policies. International Journal of Production Economics, 30-31, 167--178.

KADIPASAOGLU S.N., SRIDHARAN V. (1995) Alternative approaches for reducing schedule instability in multi-stage manufacturing under demand uncertainty. Journal of Operations Management, 13, 193--211.

KIMMS A. (1998) Stability measures for rolling schedules with applications to capacity expansion planning, master production scheduling and lot sizing. International Journal of Management Science, 26 (3), 355--366.

KLEIJNEN J. P.C., GAURY E. (2003) Short-term robustness of production management systems: A case study. European Journal of Operational Research, 148, 452--465. 
KOLTAI T., TERLAKY T. (2000) The difference between the managerial and mathematical interpretation of sensitivity analysis results in linear programming. International Journal of Production Economics, 65, 257--274.

LEE J. H., YU Z. H. (1997) Worst-case formulations of model predictive control for systems with bounded parameters. Automatica, 33, 765--781.

LIM J. M., KIM, K. S., YUM, B. J., HWANG, H. (1996) Determination of an optimal configuration of operating policies for direct-input-output manufacturing systems using the Taguchi method. Computers Industrial Engineering, 31, 555--560.

LING R.C. (2000) For true Enterprise integration, Turn First to SOP, APICS, The performance Advantage revue.

MILLER T. (2004) A hierarchical Supply Chain Planning Approach, Business Briefing. Global Purchasing \& Supply Chain Strategies, 42--57.

MINIFIE, J.R., DAVIS R.A. (1990) “Interaction effects on MRP nervousness”. International Journal of Production Research, 28, 173--183.

MUKHOPADHYAY S.K., DWIVED Y.J. and KUMAR A. (1998) Design and implementation of an integrated Production Planning System for a pharmaceutical manufacturing concern in India. Production, Planning and Control, $9 \mathrm{n}^{\circ} 4$.

ORLICKY J. (1975) Material Requirements Planning, McGraw-Hill, London.

ORTIZ ARAYA V., THOMAS A., HUTT C. "Proposition of desegregation method for tactical robust planning”. IFAC/IEEE Conference on Management and Control of Production and Logistics 2004 - Santiago (Chili) - Nov 2004.

ROTA K., THIERRY C. and BEL G. (2002) Supply Chain Management: a supplier perspective. Production Planning and Control, 370--380.

ROY B. (1998) A missing link in OR-DA: robustness analysis. Foundations of Computing and Decision Sciences, 23, 141--160. 
SIMAMPO A., RYAN S.M. (2003) Capacity expansion for a loss system with exponential demand growth. Computers and operations research, 1525--1537.

SHANG, J. S., LI, S., and TADIKAMALLA, P. (2004) Operational design of a supply chain system using the Taguchi method, response surface methodology, simulation, and optimization. International Journal of Production research, 42(8), 3823-3849.

SHOBRYS D.E., WHITE D.C. (2000) Planning, scheduling and control systems: why can they not work together. Computers and chemical engineering journal, 24, 163--173.

SRIDHARAN S.V., LAFORGE R.L. (1990) An analysis of alternative policies to achieve schedule stability. Journal of Manufacturing and Operations Management, 3, 53--73.

STADTLER H., KILGER C. (2000) Supply Chain Management and Advanced Planning: Concepts Models, Software and Case Studies, Stadtler H., Kilger C. (ed), Springer-Verlag, Berlin.

SUHUA H. and WU M.S. (2000) Demand and cost forecast error sensitivity analyses in aggregate production planning by possibilistic linear programming models. Journal of Intelligent Manufacturing, 11 (4), 355--364.

TAGUCHI G. (1987) Orthogonal arrays and linear graph, American Supplier Institute press. TOLY C. (2003) A fuzzy mid-term single-fab production planning model. Journal of Intelligent Manufacturing, 14 (3)-(4), 273--285.

VOLLMANN T.E., BERRY W.L., WHYBARK D.C. (1997) Manufacturing planning and control systems, 4th ed., New York et al.

YANO C.A., CARLSON R.C. (1987) Interaction between frequency of rescheduling and the role of safety stock in material requirements planning systems. International Journal of Production Research, 25, 221--232.

ZÄPFEL G. (1998) Customer-order-driven production: an economical concept for responding to demand uncertainty. International Journal of Production Economics, 56/57, 699--709. 
ZHAO X., LEE T.S. (1993) Freezing the master production schedule for material requirements planning systems under demand uncertainty. Journal of Operations Management, 11 (2), 185--205. 


\section{Tables}

\begin{tabular}{|c|c|c|c|c|c|c|c|c|c|c|c|c|}
\hline & 1 & 2 & 3 & 4 & 5 & 6 & 7 & 8 & 9 & 10 & 11 & 12 \\
\hline Stock costs & 20 & 20 & 20 & 20 & 20 & 20 & 20 & 20 & 20 & 20 & 20 & 20 \\
\hline Costs of delays & 60 & 60 & 60 & 60 & 60 & 60 & 60 & 60 & 60 & 60 & 60 & 60 \\
\hline Costs of hiring new employees & 400 & 400 & 400 & 400 & 400 & 400 & 400 & 400 & 400 & 400 & 400 & 400 \\
\hline Costs of redundancies & 500 & 500 & 500 & 500 & 500 & 500 & 500 & 500 & 500 & 500 & 500 & 500 \\
\hline Costs of operators & 1600 & 1600 & 1600 & 1600 & 1600 & 1600 & 1600 & 1600 & 1600 & 1600 & 1600 & 1600 \\
\hline Costs of overtime & 15 & 15 & 15 & 15 & 15 & 15 & 15 & 15 & 15 & 15 & 15 & 15 \\
\hline Procurement costs of raw materials & 200 & 200 & 200 & 200 & 200 & 200 & 200 & 200 & 200 & 200 & 200 & 200 \\
\hline Costs of stocks of raw materials & 10 & 10 & 10 & 10 & 10 & 10 & 10 & 10 & 10 & 10 & 10 & 10 \\
\hline Cancellation costs & 60 & 30 & 0 & 0 & 0 & 0 & 0 & 0 & 0 & 0 & 0 & 0 \\
\hline Express costs & 60 & 30 & 0 & 0 & 0 & 0 & 0 & 0 & 0 & 0 & 0 & 0 \\
\hline Forecast Ft & 85 & 20 & 30 & 30 & 15 & 20 & 20 & 20 & 60 & 90 & 75 & 55 \\
\hline
\end{tabular}

Table 1: Data of Tactical Plan Costs

Initial Stock (unit) $\mathrm{t}=0$ :

Initial number of employees $\mathrm{t}=0$ :

Opening hours /month/person :

Units/month/person

Maximum overtime hours/month/pers :

Manufacturing time (hours/unit) :

alpha

Initial stock of raw materials (unit) $t=0$ :

\begin{tabular}{|r|}
\hline 0 \\
5 \\
160 \\
8 \\
20 \\
20 \\
0,3 \\
0 \\
\hline
\end{tabular}

Final stock :

Final number of employees :

machine capacity (unit/month) :

Maximum stock (unit):

Table 2: Tactical Plan Data 


\begin{tabular}{|l|l|l|l|l|l|}
\hline $\begin{array}{l}\ldots . . . \text { : best approach } \\
\text { a.. second best } \\
\text { approach }\end{array}$ & Basic case & $\begin{array}{l}\text { Planning time } \\
\text { fences } \\
\text { horizon) }\end{array}$ & $\begin{array}{l}\text { Management by } \\
\text { exception } \\
\text { (Dynamic } \\
\text { Horizon) }\end{array}$ & $\begin{array}{l}\text { Management } \\
\text { by reference } \\
\text { plan }\end{array}$ \\
\hline Mean total costs & 250562 & 241715 & 249027 & 245677 \\
\hline Standard deviation & 32391 & 36673 & 28706 & 31820 \\
\hline Range of variation & 229150 & 243804 & 231511 & 219236 \\
\hline Mean service level & $62 \%$ & $51 \%$ & $78 \%$ & $71 \%$ \\
\hline Stability & 11 & 0 & $1 *$ & 2 \\
\hline
\end{tabular}

Table 3: Summary of the Management Methods for all of the Simulations 


\section{Illustrations}

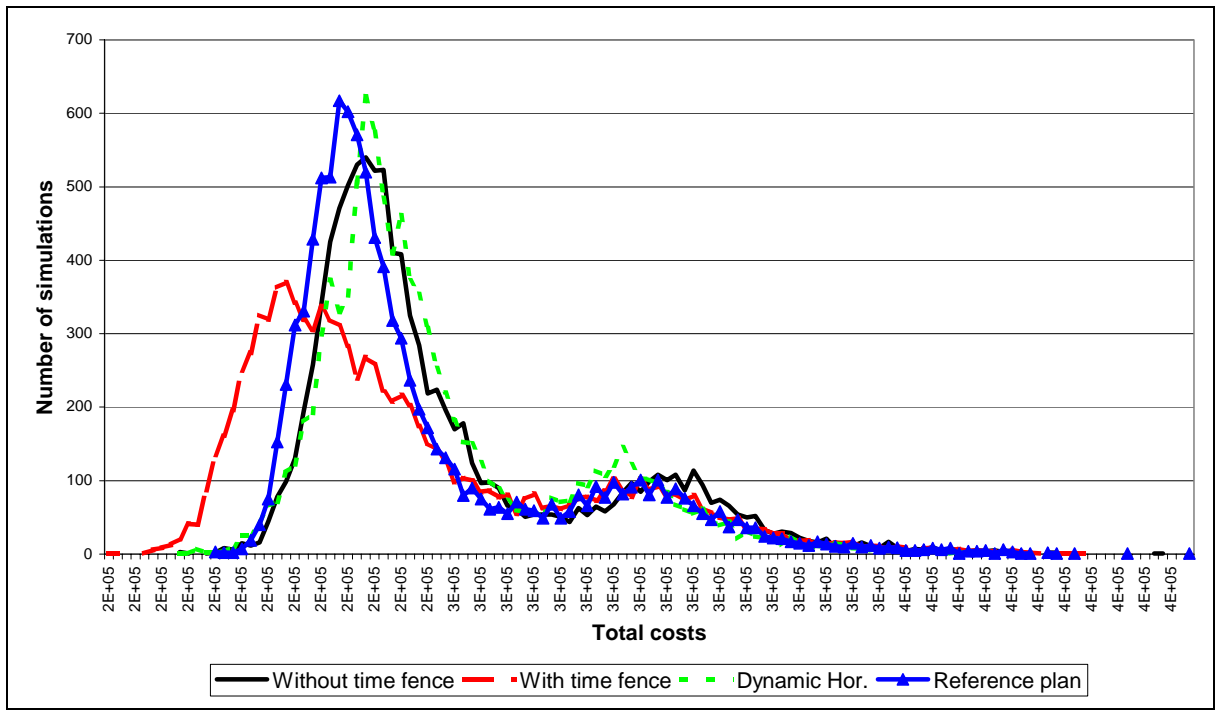

Figure 1: Distribution of Costs of the Different Management Methods

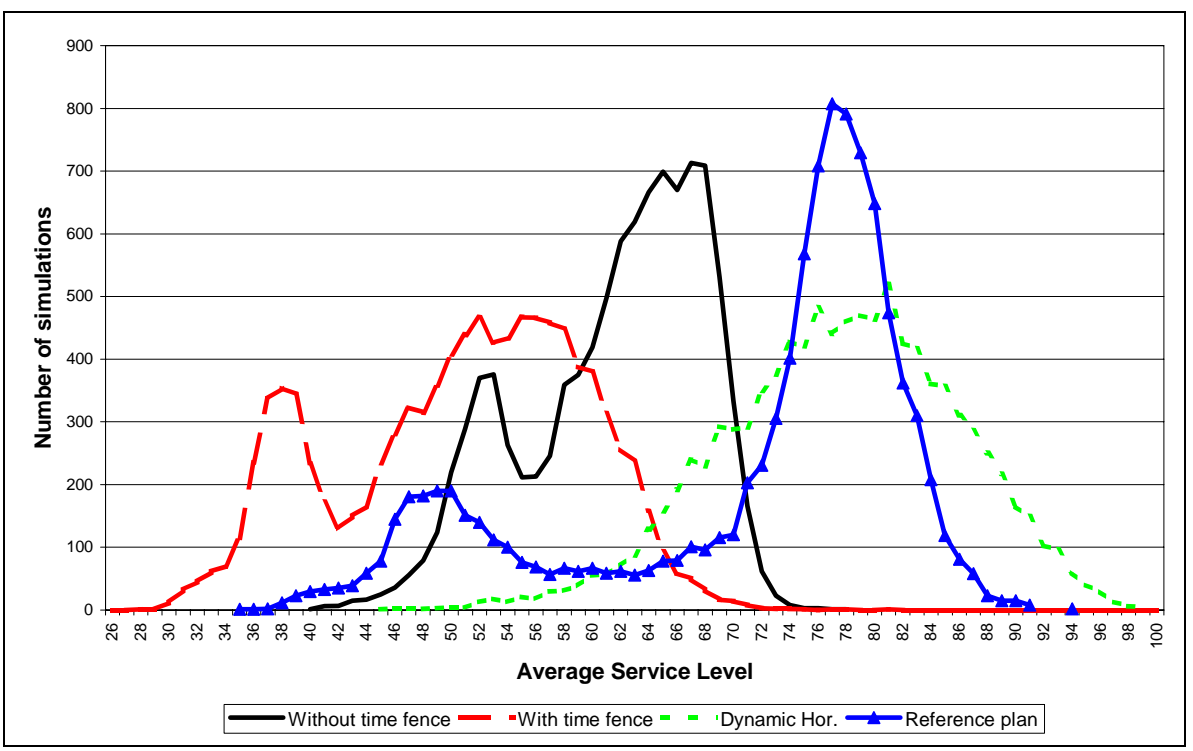

Figure 2: Service levels of the Various Management Methods 


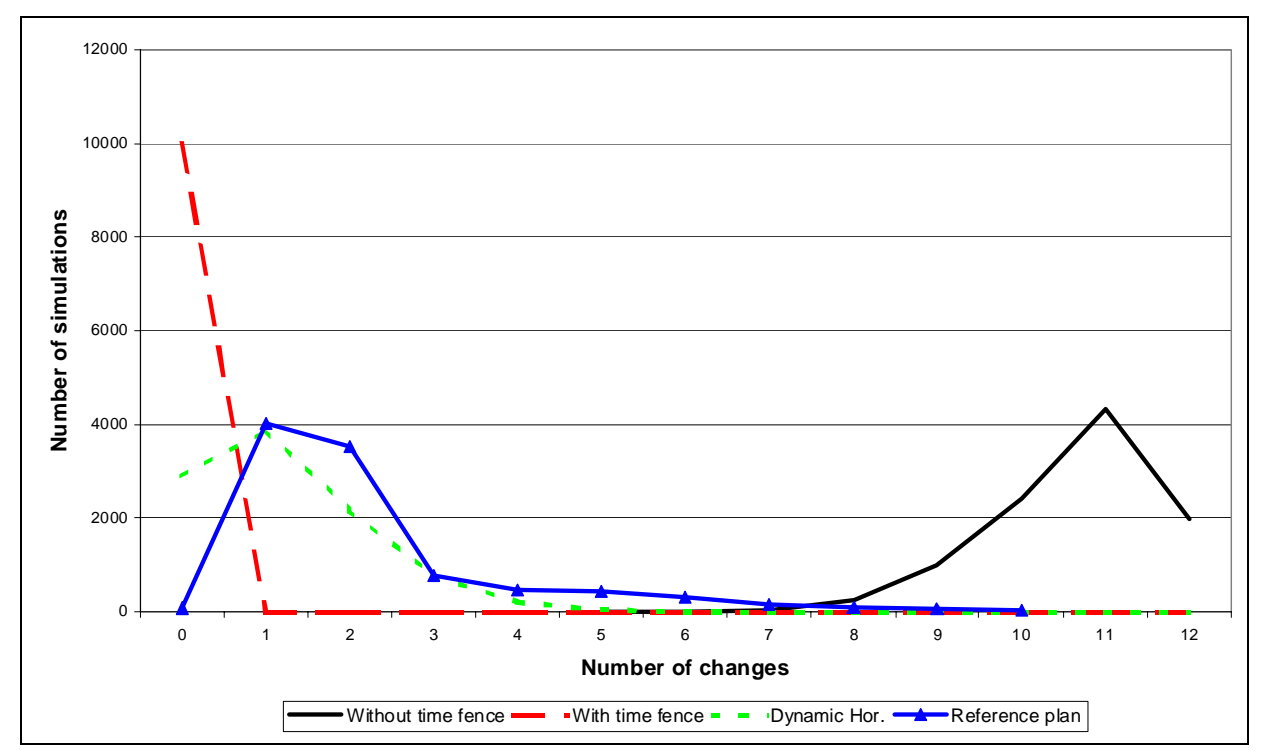

Figure 3: Indicator of Stability of the Management Methods 